\title{
Novel Treatment for Graft-versus-Host Disease
}

\author{
Yoshihiro Inamoto', Robert Zeiser², Godfrey Chi-Fung Chan ${ }^{3,4,5}$
}

'Department of Hematopoietic Stem Cell Transplantation, National Cancer Center Hospital, Tokyo, Japan, ${ }^{2}$ Department of Medicine I, Medical Center - University of Freiburg, Faculty of Medicine, Albert Ludwigs University (ALU), Freiburg, Germany, ${ }^{3}$ Department of Paediatrics and Adolescent Medicine, LKS Faculty of Medicine, The University of Hong Kong, ${ }^{4}$ Department of Paediatrics and Adolescent Medicine, Hong Kong Children's Hospital, ${ }^{5}$ Department of Paediatrics and Adolescent Medicine, HKU-Shenzhen Hospital

\section{Abstract}

Allogeneic hematopoietic cell transplantation is a curative therapy for a variety of hematological diseases, but its success is hampered by acute and chronic graft-versus-host disease (GvHD). In the last five years, multiple novel therapeutic approaches for GvHD have entered the arena. The National Institutes of Health consensus criteria for chronic GVHD have set standards for designing and reporting clinical trials, and preclinical experiments of chronic GvHD have revealed the central roles of regulatory T cells, B-cell signaling, Th17 cells, Tc17 cells, follicular helper T cells, follicular regulatory T cells, and fibrosis-promoting factors. These scientific efforts and the resulting clinical studies led to the approval of ibrutinib, belumosudil and ruxolitinib for the treatment of refractory chronic GvHD. Recently, large randomized phase III trials showed that ruxolitinib was superior to the best available therapy for glucocorticoid-refractory acute GvHD (REACH2 trial) and glucocorticoid-refractory chronic GvHD (REACH3 trial). Furthermore, novel regenerative approaches, including IL-22, R-spondin, and glucogon-like peptide-2, and cellular therapies, such as the transfer of mesenchymal stem cells and regulatory $T$ cells, are under intensive investigation. GvHD prevention using abatacept, dipeptidyl peptidase 4 inhibition, and post-transplant cyclophosphamide are also promising strategies that require further evaluation. In this article, we summarize the emerging knowledge of acute GvHD, chronic GvHD, and preclinical and clinical data of mesenchymal stem cells as GvHD therapy. In the next five years, basic and clinical studies will further advance the field, and dramatic changes in GvHD management will be encountered.

Key words graft-versus-host disease, mesenchymal stem cells, novel drugs, GVHD treatment, regenerative approaches

Submitted October 1, 2021; Accepted October 16, 2021; Published online November 25, 2021; Issued online November 25, 2021

Correspondence: Yoshihiro Inamoto, Department of Hematopoietic Stem Cell Transplantation, National Cancer Center Hospital, 5-1-1, Tsukiji, Chuo-ku, Tokyo, 104-0045, Japan, E-mail: yinamoto@ncc.go.jp

\section{Introduction}

Allogeneic hematopoietic cell transplantation (alloHCT) is a curative therapy for a variety of conditions, including hematologic disorders, metabolic storage diseases, immune deficiencies, and hematological malignancies. Acute and chronic graft-versus-host diseases (GvHDs) are major barriers that need to be overcome to make allo-HCT safer ${ }^{1,2}$. In the last five years, multiple novel therapeutic approaches for GvHD have been explored (Table 1). In this study, we summarize emerging knowledge of acute GvHD (aGvHD), chronic GvHD (cGvHD), and preclinical and clinical data on using mesenchymal stem cells (MSCs) as GvHD therapy.

\section{Novel Drugs for GvHD}

The conditioning regimen before allo-HCT causes tissue damage and activation of host antigen presenting cells (APCs) and neutrophils in the target organs of GvHD and secondary lymphoid organs. ${ }^{3}$ Activation occurs via bacterial components and danger signals, such as ATP $^{4}$ and uric acid, ${ }^{5}$ that can activate the NLRP3 inflammasome, which has been shown to play a role in aGvHD. Novel NLRP3 inhibitors that counteract in- 
Table 1. Novel therapeutic approaches for GvHD

\begin{tabular}{ll}
\hline Targeted mechanism & Drug \\
\hline Activation of host APC and neutrophils & NLRP3 inhibitors \\
\hline Influx of bacteria & PNAG or vaccination against PNAG \\
\hline Tissue regeneration & KGF, IL-7, R-spondin, GLP-2 \\
\hline JAK1/2 & Ruxolitinib, Itacitinib, Baricitinib \\
\hline TNF production in CNS GVHD & Takinib \\
\hline DPP-4 & Sitagliptin \\
\hline Interaction of CD28 and CD80/CD8 & Abatacept \\
\hline B cell/BCR signaling & Ibrutinib, Acalabrutinib, Ofatumumab, Obinutuzumab \\
\hline Proteasome & Ixazomib \\
\hline Monocyte/macrophage & Axatilimab \\
\hline Hedgehog & Glasdegib \\
ROCK2 & Belumosudil \\
Pro-inflammatory immune cells & Mesenchymal stem cells \\
\hline APC, antigen presenting cell; NLRP3, NLR family pyrin domain containing 3; PNAG, polyclonal anti- \\
bodies to poly-N-acetylglucosamine; KGF, keratinocyte growth factor; GLP-2, glucagon-like peptide-2; \\
TNF, tumor necrosis factor; CNS, central nervous system; DPP-4, dipeptidyl Peptidase-4; ROCK2, \\
Rho-associated coiled-coil kinase 2.
\end{tabular}

flammation ${ }^{6}$ could become new therapeutic interventions for aGvHD. A second early step in the pathogenesis of aGVHD is the activation of monocytes ${ }^{7}$ and neutrophil granulocytes ${ }^{3}$ which cause tissue damage and antigen presentation ${ }^{8}$. Treatment with polyclonal antibodies targeting poly-N-acetylglucosamine (PNAG) or vaccination against PNAG reduced the influx of bacteria, decreased neutrophil recruitment, and improved aGVHD in mice. Furthermore, APCs express major histocompatibility complex (MHC) and minor histocompatibility molecules that are recognized by the Tcell receptor of donor T-cells. The classical prevention and treatment of GvHD includes different immunosuppressive approaches, such as use of calcineurin inhibitors, antimetabolites, mTOR inhibitors, and corticosteroids; extracorporeal photopheresis (ECP), and others ${ }^{1,2}$. These immunosuppressive mechanisms also suppress the beneficial anti-leukemic immune response and antiinfectious immunity.

Novel strategies to reduce immunosuppressive therapy after allo-HCT include regenerative approaches, such as $\mathrm{KGF}^{10}$, interleukin (IL)- $7^{11}$, R-spondin ${ }^{12,}{ }^{13}$, and GLP- $2^{14}$. These tissue regenerative approaches act on the intestinal tract through their protective impact on Paneth cells and intestinal stem cells. Another important novel concept for aGvHD treatment is JAK1/2 inhibition with ruxolitinib, which was successfully translated from the mouse model ${ }^{15}$ into clinical application ${ }^{16,17}$. In a retrospective study, 95 patients received ruxolitinib as a salvage therapy for steroid-refractory $\mathrm{GvHD}^{18}$. The overall response rate (ORR) was $81.5 \%$, and patients with severe intestinal or skin GvHD showed an impressive response to treatment. The reduction of inflammatory disease was linked to decreased serum proinflam- matory cytokine levels and reduced numbers of activated T-cells. Although cytopenia was observed in some patients, ruxolitinib was found to be safe and well tolerated. The next step after the successful phase III trials may be a combination with other approaches; for instance, the combination of ruxolitinib with ECP was shown to have activity against $\mathrm{cGvHD}^{18}$. Novel approaches in GvHD could include kinase inhibitors for kinases relevant for the disease. GvHD caused activation of microglia cells as shown by morphological changes and differential gene expression. Selective genetic ablation of TGF- $\beta$-activated kinase-1 (TAK1) or tumor necrosis factor (TNF) in microglia identified the TAK1/ TNF/MHC-II axis as a central mediator of CNSinflammation $^{19}$. The kinase inhibitor, Takinib, reduced TNF production and CNS GvHD.

Dipeptidyl peptidase-4 (DPP-4; also known as CD26) is a homodimeric type-II transmembrane receptor with serine peptidase activity in its extracellular domain. This molecule is expressed in hematopoietic cells, endothelial cells, and epithelial cells, and is soluble and enzymatically active in the blood. DPP-4 can be inhibited by the selective inhibitor sitagliptin. Prophylaxis treatment with sitagliptin showed a reduced incidence of aGvHD compared to historical controls (NCT 00862719).

ECP is an immunomodulatory and immunosuppressive regimen. During ECP, PBMCs are collected by leukapheresis, treated with 8-methoxypsoralen (8MOP), exposed to UVA light, and re-infused into the patient. The application of ECP was shown to be a promising treatment strategy for aGvHD and cGvHD. Mechanistically, ECP increased FoxP3 ${ }^{+}$Tregs and IL-10 production. Tregs depend on IL-2 ${ }^{20}$ and IL-2 treatment has 
been shown to reduce cGvHD. GvHD or leukemia relapse $^{21}$ may change the metabolic activity of donor Tcells. Metabolic reprogramming of T-cells after alloHCT from fatty acid oxidation to aerobic glycolysis has been reported ${ }^{22}$. The mTOR inhibitor rapamycin reduced the glycolytic activity of donor T-cells. The role of this pathway was confirmed using Mtor-deficient T-cells as donors, whereas further detailed analysis with Raptordeficient T-cells revealed that T-cell pathogenicity is only dependent on mTORC1, but not mTORC $2^{22}$. Abatacept, known as cytotoxic T-cell-lymphocyte-4 (CTLA4)-immunoglobulin, is a fusion protein between the extracellular domain of human CTLA4 and a modified $\mathrm{Fc}$ region of human $\mathrm{IgG}$ and blocks co-stimulation of T-cells. Therefore, blocking the interaction between CD28 and CD80/CD86 is a promising option for reducing aGvHD. In a clinical trial of abatacept for aGvHD prophylaxis (NCT01012492), patients received cyclosporine/methotrexate (StdRx cohort) or abatacept (ABA cohort) as GVHD prophylaxis. Compared to the StdRx cohort, the ABA cohort showed a significant inhibition of early $\mathrm{CD}^{+}{ }^{+}$T-cell proliferation and activation, mainly affecting effector memory T-cells. The treatment had minor effects on $\mathrm{CD}^{+}{ }^{+} \mathrm{T}$-cells, suggesting that combination of therapies should be considered in future studies. GvHD analysis revealed only a low rate of aGvHD in the ABA cohort compared to the StdRx cohort, whereas all patients showed immune reconstitution. Furthermore, the investigators only reported two cases of grade II-IV aGvHD until day 100. The reconstitution of natural killer (NK) cells occurred relatively quickly upon abatacept treatment, while T-cell proliferation was reduced. Moreover, abatacept allowed longlasting immune reconstitution. When interfering with aGvHD, it is important to consider the effect of graftversus-leukemia (GVL). Recently, several measures have been studied to enhance the effects of GVL, such as kinase inhibition ${ }^{23}$, demethylation, and immune checkpoint inhibition $^{24,25}$.

\section{Update on the Treatments of cGvHD}

Approximately $40 \%$ of patients who undergo alloHCT develop cGvHD. It affects a variety of organs and causes significant morbidity and mortality. The manifestations of cGvHD resemble those of autoimmune diseases. Inflammation, cellular immunity, humoral immunity, and fibrosis have been implicated in the pathogenesis of $\mathrm{cGvHD}^{26}$. Characteristics of cGvHD may have ethnic differences. A recent study showed that the incidence of $c$ GvHD according to the National Institutes of Health criteria is lower in Japanese patients than in white patients ${ }^{27}$. Moreover, Japanese patients have more frequent involvement with eyes and liver, less frequent gastrointestinal involvement, and a greater number of involved sites. Despite the higher global score and a greater number of sites involved with cGvHD at onset in Japanese patients, the duration of immunosuppression and the probability of non-relapse mortality are similar between Japanese and white patients $^{27}$. Knowledge of such ethnic differences in the characteristics of cGvHD is important for the management of patients with cGvHD in different regions.

The NIH consensus project for cGvHD has made major contributions in the advances of the field by setting standards for designing and reporting clinical tri$\mathrm{als}^{28}$. Preclinical experiments of cGvHD have also revealed the central roles of regulatory T-cells, B-cell signaling, Th17 cells, Tc17 cells, follicular helper T cells, follicular regulatory $\mathrm{T}$ cells, and fibrosis-promoting factors $^{26}$. All these efforts led to the first approval from the Food and Drug Administration (FDA) of ibrutinib, a BTK inhibitor, for the treatment of cGvHD after failure of one or more lines of systemic treatment ${ }^{29}$. The best response rate after ibrutinib treatment for patients with cGvHD, including erythematous skin or mouth, was $67 \%$, and $71 \%$ of responders showed a sustained response for more than 20 weeks. Responses were observed across all involved organs evaluated, and most responders were able to reduce the dose of corticosteroids. Plasma levels of soluble factors associated with inflammation, fibrosis, and cGvHD significantly decreased over time with ibrutinib treatment. The most common grades 3-5 adverse events were pneumonia, fatigue, and diarrhea.

Recently, the results of three multicenter randomized trials for cGvHD treatment have been reported. The first international double-blind trial tested ibrutinib, in addition to standard corticosteroids, for the initial treatment of cGvHD in 193 patients (NCT02959944) $)^{30}$. The primary endpoint, complete, or partial response at 48 weeks did not differ statistically between the arms (prednisone + ibrutinib $41 \%$ versus prednisone + placebo $37 \%$ ). Meanwhile, based on secondary endpoints, several numerical trends of improved outcomes were noted in the experimental arm without increasing toxicities. The second international trial compared ruxolitinib, a JAK1/2 inhibitor, with the best available therapy for glucocorticoid-refractory cGvHD among 329 patients $(\text { NCT03112603) })^{31}$. The primary endpoint, the best overall response rate, was higher in ruxolitinib than the best available therapy (50\% versus $26 \%$ ), and failure-free survival was higher in ruxolitinib than the best available therapy. Anemia was a slightly frequent adverse event with ruxolitinib, while the frequencies of other adverse events were similar between the arms. Ruxolitinib was recently approved by the FDA for cGvHD after failure of one or two lines of systemic therapy in adult and pe- 
diatric patients 12 years and older. The third U.S. trial was a pivotal phase II randomized trial of belumosudil, a ROCK2 inhibitor, for cGvHD after two or more lines of treatment in 132 patients (NCT03640481) ${ }^{32}$. Two different doses of belumosudil (200 mg QD or BID) were compared. The primary endpoint, the best overall response rate, was $73 \%$ in all patients, and the rates were similar across all subgroups. Belumosudil was well tolerated with manageable adverse events, consistent with the results of a previous phase IIa dose-finding study ${ }^{33}$. Belumosudil was recently approved by the FDA for cGvHD after failure of at least two prior lines of systemic therapy.

In addition to the encouraging results from multicenter randomized trials, an increasing number of investigational agents that target different biological pathways of cGvHD are under development in clinical trials. The targeted mechanisms include JAK1/2, B cell/B cell receptor signaling, proteasome, ROCK2, regulatory $\mathrm{T}$ cells, monocytes/macrophages, co-stimulatory molecules, Hedgehog, and others. To address the challenges in a rapidly changing field, a third NIH consensus project was held in November 2020. Investigators aimed to define basic and clinical research directions that may lead to significant changes in cGvHD management over the next five years ${ }^{34-38}$. Four working groups have discussed etiology/prevention, diagnosis/preemptive therapy, initial and subsequent lines of systemic treatment, and highly morbid forms of $\mathrm{cGvHD}$, including sclerotic form, bronchiolitis obliterans, ocular GvHD, and gastrointestinal GvHD. Initial and second insults have been proposed as etiologies of cGvHD, and many emerging targets have been suggested for current and future preventive and therapeutic strategies ${ }^{34}$. Although further research on prognostic or predictive biomarkers of cGvHD is required, preemptive therapy might be possible in the near future ${ }^{35,36}$. Glucocorticoid-free treatment has been proposed as a future study design for initial treatment $^{37}$. Furthermore, researches highly prioritize the establishment of appropriate endpoints for each of the highly morbid form of cGvHD and designing novel trials using a small number of patients ${ }^{38}$.

\section{Mesenchymal Stem Cells (MSCs) as a Therapy for GvHD}

MSCs are one of the most potent immunosuppressive cells in the immune system. Their immunosuppressive functions have been extensively investigated. The mechanisms involve activation of immunotolerance cells (i.e. Treg), suppression of pro-inflammatory immune cells via direct cellular contact (i.e. T lymphocytes, dendritic cells, and NK cells), and secretion of immunosuppressive soluble factors (i.e. IL-10). However, the immunosuppressive function of MSCs can be altered in several situations, and MSCs may be converted as pro-inflammatory cells ${ }^{39}$. With such a wide spectrum of immunomodulatory capacity, it is natural to consider MSCs as a potential strategy for cellular therapy of autoimmune or immune-dysregulated diseases ${ }^{40,}{ }^{41}$. GvHD is caused by immune dysregulation after allo$\mathrm{HCT}^{42}$.

\section{Pre-clinical in vitro evidence}

MSCs can be isolated from a variety of tissues, such as the bone marrow, skeletal muscles, dental pulp, bone, umbilical cord, and adipose tissue. MSCs have immune privilege status due to low expression of MHC-II and costimulatory molecules on their cell surface. However, this status may change once MSCs differentiate into more lineage-specific cells, or they are exposed under the influence of interferon or infec$\operatorname{tion}^{43,44}$. MSCs can interact with a wide variety of immune cells through direct cell-to-cell interactions and secretion of soluble factors. MSCs inhibit the proliferation of most immune cells in vitro, including $\mathrm{B}$ cells, $\mathrm{T}$ cells, NK cells, and dendritic cells (DCs), producing a condition known as "division arrest anergy" ${ }^{45}$. In addition, MSCs can alter a variety of functions exerted by the immune cells, including cytokine release, cellular cytotoxicity by $\mathrm{T}$ - and NK cells, maturation and antibody secretion by B cells; and differentiation, maturation, and antigen presentation of DCs. It is thought that MSCs must be stimulated to execute their immunomodulatory function ${ }^{46}$. Among different stimulations, the release of pro-inflammatory cytokines, such as TNF- $\alpha$ and interferon-gamma may be the primary factors in the activation of MSCs. Furthermore, MSCs can recruit immune-tolerant Tregs to the lymphoid organs and graft tissues. Investigations are still ongoing to elucidate the exact mechanisms and molecules involved in the immunosuppressive effect of MSCs.

\section{Pre-clinical in vivo evidence}

In search of the possible immunomodulatory mechanisms in vivo, many molecules, such as prostaglandin $\mathrm{E}$ 2, TGF- $\beta$, IL-6 and IL-10, matrix metalloproteinases, indoleamine-2,3-dioxygenase, and nitric oxide ${ }^{30}$ have all been identified in different experimental models. In vivo studies have shown conflicting results regarding the immunomodulatory properties of MSCs in the HCT setting. However, studies have determined the efficacy of MSC therapy in different scenarios. Some studies have explored the therapeutic potential of MSCs in preventing or treating graft rejection ${ }^{47}$, while others have focused on the effects of MSCs in preventing or treating GvHD. Subsequent Phase I, II, and III clinical trials were conducted. Clinical conditions include a variety of 
autoimmune diseases, such as inflammatory bowel diseases, lupus erythematosus, rheumatoid arthritis, and even type 1 diabetes mellitus ${ }^{48-51}$. In organ transplant settings, MSCs have been used to prevent rejection of solid organ allografts ${ }^{52}$.

Among all potential applications, GvHD in the HCT setting is the first to be evaluated. GvHD can be classified according to the temporal profile or underlying mechanisms of either aGvHD or cGvHD. The pathogenesis of aGvHD is related to alloreactive T-cells, especially cytotoxic T-cells. However, cGvHD is believed to be related with altered $\mathrm{B}$ cell subpopulations, aberrant $\mathrm{B}$ cell signaling pathways, T/B cell interactions, and production of autoantibodies ${ }^{53}$. The animal model for these two types of GvHD also varied. More preclinical data on the use of MSCs for aGvHD are available compared to cGvHD.

Together with another research group, we have found that systemic infusion of human MSCs can alleviate the severity of murine aGvHD manifestation and improve survival $^{54}$. We utilized irradiated $\mathrm{BALB} / \mathrm{c}$ host mice to receive $\mathrm{C} 57 \mathrm{BL} / 6$ donor $\mathrm{T}$-cell-depleted bone marrow with or without donor CD4+ T lymphocytes as controls. Human-derived MSCs were administered via tail vein injection. We found that both survival rate and GvHD score improved in the MSC-treated mice. The levels of pro-inflammatory cytokines/chemokines, including RANTES, CCL3, CXCL9, CCR5, and CXCR3, as well as donor T-cell alloreactivity, decreased after transplantation. Homing of MSCs into the lymphoid organs and target tissues was also observed.

However, murine MSCs are not exactly the same as human MSCs; murine MSCs have a unique marker, Sca-1, and can be isolated and quantified easily. Moreover, their immune pathways and expression of immune mediators are not the same as those in the human immune system ${ }^{55}$. Therefore, the direct translation of the data into a human setting must be interpreted with caution.

\section{Clinical evidence}

MSCs have been extensively tested in steroidresistant GvHD, a devastating condition that may occur in acute or chronic forms following allo-HCT. Results from an early single-center clinical trial showed that refractory aGvHD had a favorable response to allogeneic MSC treatment ${ }^{56}$. However, subsequent multi-center phase III trials failed to observe statistically significant effects of MSC treatment on patients with refractory aGvHD. Unfortunately, the results were only presented in international meetings without publication, so the results were not included in the subsequent metaanalysis $^{57}$. Thus, the optimal method of administration of MSCs, such as the appropriate dosage and frequency of administration, remains undetermined. In addition, due to the lack of specific biomarkers, tracking of MSC homing and engraftment in vivo is challenging. Other barriers include the sources and production of MSCs, which may have a significant impact on efficacy in $v_{i v o}{ }^{58}$. The heterogeneity of MSCs with different stromal differentiation potential and biological activities may also affect the outcome. Proper randomized clinical trials should be conducted when most of these variables are controlled.

To determine the efficacy of MSCs as a prophylaxis or treatment for GvHD after HCT, a Cochrane systematic review was performed, which included 12 completed randomized control trials with 879 participants. Seven trials used MSCs as prophylaxis, and five trials utilized MSCs as treatment. However, the major critique of the existing data was that the overall quality was low due to the suboptimal methodology adopted, including poor randomization. Therefore, the risks of performance and reporting biases were considered to be high. As mentioned in previously, the results from a large-scale multi-center randomized trial that was conducted more than a decade ago were not published, possibly because the trial failed to reach the expected outcome. Omitting such negative data may lead to a publication bias. The review also concluded that the mortality in both the prophylactic and therapeutic trials remained similar between the MSC-treated group and the control group. However, there were no significant side effects reported in the therapeutic trials, and the risk of malignant disease relapse did not increase. MSCs were well tolerated, but there was no health-related quality of life assessment found in these trials to support this observation. Among the prophylactic trials, it was shown that MSCs may reduce the risk of $\mathrm{cGvHD}$ (relative risk [RR]: 0.66, 95\% Confidence Interval [CI]: 0.49-0.89), but not the risk of aGvHD. For the GvHD therapeutic trials, there was no evidence of any differences in the outcomes $^{59}$.

Another recently published meta-analysis presented a different conclusion. This study included nonrandomized trials and analyzed 16 prophylactic and 35 therapeutic studies. Six hundred fifty-four patients received MSCs for GvHD prophylaxis, and there was a $17 \%$ higher overall survival (95\% CI: 1.02-1.33) with a lower incidence of grade $4 \mathrm{aGvHD}$ (RR: $0.22 ; 95 \% \mathrm{CI}$ : 0.06-0.81) and cGvHD (RR: 0.64; 95\% CI: 0.47-0.88) than the controls. If both the prophylaxis and treatment group of $>1,600$ patients were observed, the overall survival rate of patients with aGvHD treated with MSCs was also significantly higher than that of the control group ( $\mathrm{p}=0.0214)$, with a survival rate of approximately 50\% (95\% CI: 0.41-0.59). The response rate of patients with aGvHD to MSC treatment was approxi- 
mately $67 \%$, and $39 \%$ had a complete response. The skin had the best response rate among different organs affected by aGvHD. Twenty-two percent of patients with aGvHD infused with MSCs developed cGvHD, but $64 \%$ of this group survived. This meta-analysis suggests that allogeneic MSCs can serve as part of our armamentarium against GvHD, especially when GvHD is refractory to conventional therapies such as pulse steroids.

To resolve the conflicting results of these two metaanalyses, good quality clinical trials must be conducted $^{60}$. In addition, a number of confounding factors must be properly standardized and controlled. We propose several novel solutions to standardize the production of clinical-grade MSCs.

\section{Possible causes of suboptimal clinical trial results and the possible solution to overcome these hin- drances}

We found that even for bone marrow-derived MSCs, several factors, such as the donor's health status and orders of aspiration, can affect the harvest yield. The optimal bone marrow samples for MSC collection should be obtained earlier in the process of harvesting ${ }^{61}$. We also noticed that MSCs from patients suffering from various diseases may not behave normally (e.g. MSCs from lupus patients) ${ }^{62}$. Interestingly, MSCs from patients with acute leukemia or immune thrombocytopenia purpura regain normal phenotype after several passages of ex vivo culture, suggesting that the influence of extrinsic factors on MSCs is transient and may not have a permanent impact.

It has been argued that even though MSCs are considered as "stem cells," they have finite expansion potential in vitro and will become senescent after a limited number of culture passages. This becomes a major quality control issue for clinical applications because one may encounter possible batch-by-batch variations. Thus, acquisition of accreditation from healthcare authorities will be a challenge. To overcome this issue of cell heterogeneity and the limited number of passage effects, we converted selected MSCs into induced pluripotent stem cells and then differentiated them back into MSCs. Such an approach can produce a relatively unlimited supply of homogeneous MSCs of the same origin. Quality control problems can then be overcome.

Several studies have proposed that MSCs from different tissues may differ in terms of their proliferative capacity and immunomodulatory function. However, none of these studies directly compared the three types of MSCs. We confirmed this observation by comparing MSCs from adipose tissue, umbilical cord, and bone marrow using in vitro and in vivo models. We found that adipose tissue-derived MSCs proliferated much bet- ter than bone marrow-or umbilical cord-derived MSCs in both in vitro and in vivo models. We then explored possible mechanisms to elucidate such variation. By comparing the RNAseq profiles of MSCs derived from the three tissues, we identified potential unique immune molecules involved. Immunological tests confirmed that association with and blocking of this molecule would take away the proliferative and immunomodulatory advantages of MSCs. Furthermore, we discovered some existing drugs that can enhance the expression of this molecule and render MSCs more immunosuppressive. Thus, we can manipulate the immune potential of MSCs in the future by using extrinsic factors to maximize the clinical benefit of this approach.

We also explored the use of MSC-derived exosomes as a substitute for MSCs to improve product consistency $^{63}$. Exosomes contain a wide spectrum of biologically active factors, such as cytokines, growth factors, and miRNAs. By conducting in vitro and in vivo experiments, we found that exosomes derived from MSCs are as effective as MSCs in treating lupus mice. We also identified some possible immunosuppressive molecules involved.

Lastly, to address the problem of product scale-up, in collaboration with the bioengineering team of Oxford University (UK), we invented an ex vivo automated oscillating cylinder culture system. This system utilizes microbeads as a culture-adherent surface that can improve the yield of MSCs. We found that it can generate much more cells than the microfiber culturing system since it provides a much larger surface area for attachment and allows the MSCs to cluster together even in the early culturing period. It is also easier to harvest from the microbeads because it tends to spread on the adherence surface once they are resting on a culture plate. All these quality improvement steps help to move MSC therapy back to the bedside again with much more consistency and efficiency.

\section{Conclusion}

New rational therapeutic concepts for GvHD should not only inhibit the inflammatory immune response, but also modulate the immune reaction to preserve graftversus-tumor effects. Here, we have discussed recently developed concepts to modulate the immune response after allo-HCT to prevent life-threatening GvHD. In the next five years, basic and clinical research will further advance the field, and dramatic changes in GvHD management will be encountered.

\section{Author Contributions}

Y.I., R.Z., and G.CF. C wrote the article. 


\section{Conflicts of Interest}

Y.I. received honoraria from Novartis, Janssen, and Meiji Seika Pharma; R.Z. received honoraria from Novartis, Incyte and Mallinckrodt, and G.C.F.C. declare no conflicts of interest.

\section{References}

1. Zeiser R, Blazar, B.R. Acute Graft-versus-host disease Biologic process, prevention, and therapy. $\mathrm{N}$ Eng $\mathrm{J}$ Med. 2017; 377: 2167-79.

2. Zeiser R, Blazar, B.R. Pathophysiology of Chronic Graftversus-Host Disease and Therapeutic Targets. N Eng J Med. 2017; 377: 2565-79.

3. Schwab L, Goroncy L, Palaniyandi S, Gautam S, Triantafyllopoulou A, Mocsai A, et al. Neutrophil granulocytes recruited upon translocation of intestinal bacteria enhance graft-versus-host disease via tissue damage. Nat Med. 2014; 20: $648-54$

4. Wilhelm K, Ganesan J., Müller T., Dürr C., Grimm, M., Beilhack, A., et al. Graft-versus-host disease enhanced by extracellular adenosine triphosphate activating P2X7R. Nature Medicine. 2010; 12: 1434-8.

5. Jankovic D, Ganesan, J., Bscheider, M., Stickel, N., Weber, F., Guarda, et al. The Nlrp3-inflammasome regulates acute graft-versus-host disease. J Exp Med. 2013; 210: 1899-910.

6. Hamarsheh S, Osswald, L., Saller, B.S., Unger S, De Feo D, Vinnakota JM, et al. Oncogenic KrasG12D causes myeloproliferation via NLRP3 inflammasome activation. Nat Commun. 2020; 11: 1659.

7. Klämbt V, Wohlfeil SA, Schwab L, Hülsdünker J, Ayata K, Apostolova P, et al. A Novel Function for P2Y2 in Myeloid Recipient-Derived Cells during Graft-versus-Host Disease. J Immunol. 2015; 195: 5795-804.

8. Hülsdünker J, Ottmüller KJ, Neeff HP, Koyama M, Gao Z, Thomas OS, et al. Neutrophils provide cellular communication between ileum and mesenteric lymph nodes at graftversus-host disease onset. Blood. 2018; 131: 1858-69.

9. Hülsdünker J, Thomas OS, Haring E, Unger S, Gonzalo Núñez N, Tugues $\mathrm{S}$, et al. Immunization against poly-Nacetylglucosamine reduces neutrophil activation and GVHD while sparing microbial diversity. Proc Natl Acad Sci U S A. 2019; 116: 20700-6.

10. Min D, Panoskaltsis-Mortari A, Kuro-O M, Holländer GA, Blazar BR, Weinberg KI. Sustained thymopoiesis and improvement in functional immunity induced by exogenous KGF administration in murine models of aging. Blood. 2007; 109: 2529-37.

11. Alpdogan O, Muriglan S J, Eng JM, Willis LM, Greenberg AS, Kappel BJ, et al. IL-7 enhances peripheral T-cell reconstitution after allogeneic hematopoietic stem cell transplantation. J Clin Invest. 2003; 112: 1095-107.

12. Takashima S, Kadowaki M, Aoyama K, Koyama M, Oshima $\mathrm{T}$, Tomizuka $\mathrm{K}$, et al. The Wnt agonist $\mathrm{R}$-spondin 1 regulates systemic graft-versus-host disease by protecting intestinal stem cells. J Exp Med. 2011; 208: 285-94.

13. Hayase E, Hashimoto D, Nakamura K, Noizat C, Ogasawara $\mathrm{R}$, Takahashi S, et al. R-Spondin1 expands Paneth cells and prevents dysbiosis induced by graft-versus-host disease. J
Exp Med. 2017; 214: 3507-18.

14. Norona J, Apostolova P, Schmidt D, Ihlemann R, Reischmann N, Taylor G, et al. Glucagon-like peptide 2 for intestinal stem cell and Paneth cell repair during graft-versushost disease in mice and humans. Blood. 2020; 136: 144255 .

15. Spoerl S, Mathew NR, Bscheider M, Schmitt-Graeff A, Chen S, Mueller T, et al. Activity of therapeutic JAK 1/2 blockade in graft-versus-host disease. Blood. 2014; 123: 3832-42.

16. Zeiser R, von Bubnoff N, Butler J, Mohty M, Niederwieser $\mathrm{D}$, Or R, et al. Ruxolitinib for Glucocorticoid-Refractory Acute Graft-versus-Host Disease. N Eng J Med. 2020; 382: 1800-10.

17. Zeiser R, Burchert A, Lengerke C, Verbeek M, Maas-Bauer $\mathrm{K}$, Metzelder SK, et al. Ruxolitinib in corticosteroidrefractory graft-versus-host disease after allogeneic stem cell transplantation: a multi-center survey. Leukemia. 2015; 29: 2062-8.

18. Maas-Bauer K, Kiote-Schmidt C, Bertz H, Apostolova P, Wäsch R, Ihorst $\mathrm{G}$, et al. Ruxolitinib-ECP combination treatment for refractory severe chronic graft-versus-host disease. Bone Marrow Transplant. 2020; 56: 909-16.

19. Mathew NR, Vinnakota JM, Apostolova P, Erny D, Hamarsheh S, Andrieux G, et al. Graft-versus-host disease of the CNS is mediated by TNF upregulation in microglia. J Clin Invest. 2020; 130: 1315-29.

20. Zeiser R, Nguyen VH, Beilhack A, Buess M, Schulz S, Baker $\mathrm{J}$, et al. Inhibition of $\mathrm{CD} 4+\mathrm{CD} 25+$ regulatory $\mathrm{T}$ cell function by calcineurin dependent interleukin-2 production. Blood. 2006; 108: 390-9.

21. Uhl FM, Chen S, O'Sullivan D, Edwards-Hicks J, Richter $\mathrm{G}$, Haring $\mathrm{E}$, et al. Metabolic reprogramming of donor $\mathrm{T}$ cells enhances graft-versus-leukemia effects in mice and humans. Sci Transl Med. 2020; 12: eabb8969.

22. Nguyen HD, Chatterjee S, Haarberg KM, Wu Y, Bastian D, Heinrichs J, et al. Metabolic reprogramming of alloantigenactivated $\mathrm{T}$ cells after hematopoietic cell transplantation. J Clin Invest. 2016; 126: 1337-52

23. Mathew NR, Baumgartner F, Braun L, David O'Sullivan, Thomas S, Waterhouse M, et al. Sorafenib promotes graftversus-leukemia activity in mice and humans through IL-15 production in FLT3-ITD mutant leukemia cells. Nature Medicine. 2018; 24: 282-91.

24. Zeiser R, Vago, L. Mechanisms of immune escape after allogeneic hematopoietic cell transplantation. Blood. 2019; 133: $1290-7$.

25. Zeiser R, Beelen DW, Bethge W, Bornhäuser M, Bug G, Burchert A, et al. Biology-Driven Approaches to Prevent and Treat Relapse of Myeloid Neoplasia after Allogeneic Hematopoietic Stem Cell Transplantation. Biol Blood Marrow Transplant. 2019; 25: 128-40.

26. Cooke KR, Luznik L, Sarantopoulos S, Hakim FT, Jagasia M, Fowler DH, et al. The Biology of Chronic Graft-versusHost Disease: A Task Force Report from the National Institutes of Health Consensus Development Project on Criteria for Clinical Trials in Chronic Graft-versus-Host Disease. Biol Blood Marrow Transplant. 2017; 23: 211-34.

27. Inamoto $\mathrm{Y}$, White $\mathrm{J}$, Ito $\mathrm{R}$, Martin PJ, Fatobene G, Ito A, et al. Comparison of characteristics and outcomes of late acute and NIH chronic GVHD between Japanese and white pa- 
tients. Blood Adv. 2019; 3: 2764-77.

28. Filipovich AH, Weisdorf D, Pavletic S, Socie G, Wingard JR, Lee SJ, et al. National Institutes of Health consensus development project on criteria for clinical trials in chronic graft-versus-host disease: I. Diagnosis and staging working group report. Biol Blood Marrow Transplant. 2005; 11: 94556.

29. Miklos D, Cutler CS, Arora M, Waller EK, Jagasia M, Pusic I, et al. Ibrutinib for chronic graft-versus-host disease after failure of prior therapy. Blood. 2017; 130: 2243-50.

30. Miklos D, Zaid MA, Cooney JP, Albring J, Flowers M, Skarbnik AP, et al. Ibrutinib vs placebo in combination with corticosteroids in patients with new-onset chronic graftversus-host disease (CGVHD): Results from the randomized, double-blind phase 3 iNTEGRATE study. EHA meeting abstract. 2021: S235.

31. Zeiser R, Polverelli N, Ram R, Hashmi SK, Chakraverty R, Middeke JM, et al. Ruxolitinib for GlucocorticoidRefractory Chronic Graft-versus-Host Disease. N Engl J Med. 2021; 385: 228-38.

32. Cutler CS, Lee SJ, Arai S, Rotta M, Zoghi B, Lazaryan A, et al. Belumosudil for Chronic Graft-versus-Host Disease (cGVHD) After 2 or More Prior Lines of Therapy: The ROCKstar Study. Blood in press. 2021.

33. Jagasia M, Lazaryan A, Bachier CR, Salhotra A, Weisdorf DJ, Zoghi B, et al. ROCK2 Inhibition With Belumosudil (KD025) for the Treatment of Chronic Graft-Versus-Host Disease. J Clin Oncol. 2021; 39: 1888-98.

34. Williams KM, Inamoto Y, Im A, Hamilton B, Koreth J, Arora $\mathrm{M}$, et al. National Institutes of Health Consensus Development Project on Criteria for Clinical Trials in Chronic Graft-versus-Host Disease: I. The 2020 Etiology and Prevention Working Group Report. Transplant Cell Ther. 2021; 27: 452-66.

35. Kitko CL, Pidala J, Schoemans HM, Lawitschka A, Flowers ME, Cowen EW, et al. National Institutes of Health Consensus Development Project on Criteria for Clinical Trials in Chronic Graft-versus-Host Disease: IIa. The 2020 Clinical Implementation and Early Diagnosis Working Group Report. Transplant Cell Ther. 2021; 27: 545-57.

36. Pidala J, Kitko C, Lee SJ, Carpenter P, Cuvelier GDE, Hol$\tan \mathrm{S}$, et al. National Institutes of Health Consensus Development Project on Criteria for Clinical Trials in Chronic Graft-versus-Host Disease: IIb. The 2020 Preemptive Therapy Working Group Report. Transplant Cell Ther. 2021; 27: 632-41.

37. DeFilipp Z, Couriel DR, Lazaryan A, Bhatt VR, Buxbaum NP, Alousi AM, et al. National Institutes of Health Consensus Development Project on Criteria for Clinical Trials in Chronic Graft-versus-Host Disease: III. The 2020 Treatment of Chronic GVHD Report. Transplant Cell Ther. 2021; 27: 729-37.

38. Wolff D, Radojcic V, Lafyatis R, Cinar R, Rosenstein RK, Cowen EW, et al. National Institutes of Health Consensus Development Project on Criteria for Clinical Trials in Chronic Graft-versus-Host Disease: IV. The 2020 Highly morbid forms report. Transplant Cell Ther. 2021.

39. Tso GH, Law HK, Tu W, Chan GC, Lau YL. Phagocytosis of apoptotic cells modulates mesenchymal stem cells osteogenic differentiation to enhance IL-17 and RANKL expression on CD4+ T cells. Stem Cells. 2010; 28: 939-54.
40. Liu F, Chen H, Chen T, Lau CS, Yu FX, Chen K, et al. Immunotherapeutic effects of allogeneic mesenchymal stem cells on systemic lupus erythematosus. Lupus. 2020; 29: 872-83.

41. Ni K, Liu M, Zheng J, Wen L, Chen Q, Xiang Z, et al. PD1/PD-L1 Pathway Mediates the Alleviation of Pulmonary Fibrosis by Human Mesenchymal Stem Cells in Humanized Mice. Am J Respir Cell Mol Biol. 2018; 58: 684-95.

42. Eljarrah A, Gergues M, Pobiarzyn PW, Sandiford OA, Rameshwar P. Therapeutic Potential of Mesenchymal Stem Cells in Immune-Mediated Diseases. Adv Exp Med Biol. 2019; 1201: 93-108.

43. Chan WK, Lau AS, Li JC, Law HK, Lau YL, Chan GC. MHC expression kinetics and immunogenicity of mesenchymal stromal cells after short-term IFN-gamma challenge. Exp Hematol. 2008; 36: 1545-55.

44. Mo IF, Yip KH, Chan WK, Law HK, Lau YL, Chan GC. Prolonged exposure to bacterial toxins downregulated expression of toll-like receptors in mesenchymal stromal cellderived osteoprogenitors. BMC Cell Biol. 2008; 9: 52.

45. Glennie S, Soeiro I, Dyson PJ, Lam EW, Dazzi F. Bone marrow mesenchymal stem cells induce division arrest anergy of activated T cells. Blood. 2005; 105: 2821-7.

46. Aggarwal S, Pittenger MF. Human mesenchymal stem cells modulate allogeneic immune cell responses. Blood. 2005; 105: 1815-22.

47. Dazzi F, Ramasamy R, Glennie S, Jones SP, Roberts I. The role of mesenchymal stem cells in haemopoiesis. Blood Rev. 2006; 20: 161-71.

48. Vasudevan A, Bruining DH, Loftus EV, Jr., Faubion W, Ehman EC, Raffals L. Approach to medical therapy in perianal Crohn's disease. World J Gastroenterol. 2021; 27: 3693-704.

49. Savio-Silva C, Beyerstedt S, Soinski-Sousa PE, Casaro EB, Balby-Rocha MTA, Simplicio-Filho A, et al. Mesenchymal Stem Cell Therapy for Diabetic Kidney Disease: A Review of the Studies Using Syngeneic, Autologous, Allogeneic, and Xenogeneic Cells. Stem Cells Int. 2020; 2020: 8833725.

50. Radmanesh F, Mahmoudi M, Yazdanpanah E, Keyvani V, Kia N, Nikpoor AR, et al. The immunomodulatory effects of mesenchymal stromal cell-based therapy in human and animal models of systemic lupus erythematosus. IUBMB Life. 2020; 72: 2366-81.

51. Lopez-Santalla M, Fernandez-Perez R, Garin MI. Mesenchymal Stem/Stromal Cells for Rheumatoid Arthritis Treatment: An Update on Clinical Applications. Cells. 2020; 9: 1852.

52. De Miguel MP, Fuentes-Julian S, Blazquez-Martinez A, Pascual CY, Aller MA, Arias J, et al. Immunosuppressive properties of mesenchymal stem cells: advances and applications. Curr Mol Med. 2012; 12: 574-91.

53. Li X, Gao Q, Feng Y, Zhang X. Developing role of B cells in the pathogenesis and treatment of chronic GVHD. Br J Haematol. 2019; 184: 323-36.

54. Robles JD, Liu YP, Cao J, Xiang Z, Cai Y, Manio M, et al. Immunosuppressive mechanisms of human bone marrow derived mesenchymal stromal cells in BALB/c host graft versus host disease murine models. Exp Hematol Oncol. 2015; 4: 13.

55. Romieu-Mourez R, Coutu DL, Galipeau J. The immune plasticity of mesenchymal stromal cells from mice and men: concordances and discrepancies. Front Biosci (Elite Ed). 
2012; 4: 824-37.

56. Le Blanc K, Samuelsson H, Gustafsson B, Remberger M, Sundberg B, Arvidson J, et al. Transplantation of mesenchymal stem cells to enhance engraftment of hematopoietic stem cells. Leukemia. 2007; 21: 1733-8.

57. Taupin P. OTI-010 Osiris Therapeutics/JCR Pharmaceuticals. Curr Opin Investig Drugs. 2006; 7: 473-81.

58. Introna M, Golay J. Tolerance to Bone Marrow Transplantation: Do Mesenchymal Stromal Cells Still Have a Future for Acute or Chronic GvHD? Front Immunol. 2020; 11: 609063.

59. Fisher SA, Cutler A, Doree C, Brunskill SJ, Stanworth SJ, Navarrete C, et al. Mesenchymal stromal cells as treatment or prophylaxis for acute or chronic graft-versus-host disease in haematopoietic stem cell transplant (HSCT) recipients with a haematological condition. Cochrane Database Syst Rev. 2019; 1: CD009768.

60. Morata-Tarifa C, Macias-Sanchez MDM, Gutierrez-Pizarraya A, Sanchez-Pernaute R. Mesenchymal stromal cells for the prophylaxis and treatment of graft-versus-host disease-a meta-analysis. Stem Cell Res Ther. 2020; 11: 64.

61. Li J, Wong WH, Chan S, Chim JC, Cheung KM, Lee TL, et al. Factors affecting mesenchymal stromal cells yield from bone marrow aspiration. Chin J Cancer Res. 2011; 23: 43-8.

62. Nie Y, Lau CS, Lie AK, Chan GC, Mok MY. Defective phenotype of mesenchymal stem cells in patients with systemic lupus erythematosus. Lupus. 2010; 850-9.

63. Zhang C, Wang H, Chan GCF, Zhou Y, Lai X, Lian M. Extracellular Vesicles Derived from Human Umbilical Cord Mesenchymal Stromal Cells Protect Cardiac Cells Against Hypoxia/Reoxygenation Injury by Inhibiting Endoplasmic Reticulum Stress via Activation of the PI3K/Akt Pathway. Cell Transplant. 2020; 29: 963689720945677.

https://doi.org/10.31547/bct-2021-022

Copyright (C)2021 Asia-Pacific Blood and Marrow Transplantation Group (APBMT). This is an open access article distributed under CC BY-NC license (https://creativecommon s.org/licenses/by-nc/4.0/). 